\title{
DUAL NECKLACES OF $n$-DIMENSIONAL CUBES
}

\author{
KENNETH KALMANSON
}

\begin{abstract}
It has been shown that certain finite configurations, called dual necklaces, of euclidean spheres yield information on longest rectilinear circuits on the sphere centers. In the present paper, the existence of dual necklaces of $k n$-dimensional cubes is discussed and the values of $k$ are determined. A more general configuration of cubes, called a multidual necklace, is treated similarly.
\end{abstract}

I. Introduction. The object of this paper is to investigate the existence of geometric realizations of two extremal conditions. These conditions, given below in Definition 1, have their origin in the following "dual necklace theorem" of D. Sanders [1]: A rectilinear polygon on $r$ vertices in the (euclidean) space $E^{n}, n>1$, is longest in its covertex class if a (closed) sphere may be centered on each vertex such that each sphere intersects all others except for the two centered on the two adjacent vertices given by the polygon.

Definition 1. A dual necklace is a collection of $k(k \geqq 3)$ subsets of coordinate space $R^{n}$ such that (condition 1) each set intersects all but two others in the collection, and (condition 2) when a unique point is designated in each set as "center" and the centers of nonintersecting sets joined by straight line segments, a rectilinear polygon is formed.

It has been shown by Warren Becker that dual necklaces of $k$ spheres exist in $E^{n}, n>1$, for all $k \geqq 3$. Define the metric space $S^{n}=\left(R^{n}, s\right)$ by $s(x, y)=\max _{1 \leqq i \leqq n}\left|x_{i}-y_{i}\right|$; the metric spheres of the space $S^{n}$ are of course $n$-dimensional cubes.

II. THEOREM 1. Dual necklaces of $k$ (closed) spheres in $S^{n}$ exist for precisely $3 \leqq k \leqq 2 n+3$ if $n \geqq 3$, and for $3 \leqq k \leqq 6$ if $n=2$.

Definition 2. A multi-dual necklace is a collection of subsets of $R^{n}$ satisfying condition 1 of Definition 1.

THEOREM 2. There exist multi-dual necklaces of $k$ closed spheres in the space $S^{n}$ for precisely $3 \leqq k \leqq 4 n$.

Received by the editors November 16, 1970 .

AMS 1969 subject classifications. Primary 5240.

Key words and phrases. Dual necklace, rectilinear circuit, $n$-dimensional cube, multidual necklace, convex, centrally symmetric, $2 n$-gon.

(C) American Mathematical Society 1972 
Lemma 1. There does not exist a dual necklace of $k$ rectangular parallelopipeds (n-dimensional, with edges parallel to the coordinate axes) if $k>2 n+3$, where $n \geqq 3$, or for $k>6$, if $n=2$.

Proof. Suppose there were such a collection, $C$. Let $P_{0}$ belong to $C$ and let $P_{1}$ and $Q_{1}$ be the two members of $C$ which do not intersect $P_{0}$. Then $P_{1}$ intersects $Q_{1}$, or else we would be forced to join the centers of $P_{0}, P_{1}, Q_{1}$ to obtain a triangle. This is impossible since $n \geqq 2$. Moreover, there are additional members $P_{2}$ and $Q_{2}$ of $C$ such that $P_{2} \cap Q_{1}=\varnothing, Q_{2} \cap P_{1}=\varnothing$, but $P_{2} \cap P_{1} \neq \varnothing, Q_{2} \cap Q_{1} \neq \varnothing$ and $P_{2} \cap Q_{2} \neq \varnothing$. If this were not the case, that is, if there were a member $S$ in $C$ which intersected $P_{0}$ but neither $P_{1}$ nor $Q_{1}$ we must join the centers of $P_{0}, P_{1}, S, Q_{1}$, and $P_{0}$ in that order, obtaining a quadrilateral. Similarly, $P_{2}$ and $Q_{2}$ must intersect or we obtain a pentagon. Finally, each of the remaining members of $C$ must intersect $P_{0}, P_{1}$, and $Q_{1}$.

Let us continue by defining $P_{0}$ to be a particular member of $C$. We will denote the perpendicular projection of a member $R$ of $C$ on tr- $i$ th coordinate axis by $R^{i}, i=1, \cdots, n$. Let $P_{0}$ be any member of $C$ such that the right-hand endpoint of $P_{0}^{1}$ is minimal among all right-hand endpoints of the intervals $R^{1}$, for all $R$ in $C$. Observe that two members $P$ and $Q$ in $C$ intersect if and only if we have $P^{i} \cap Q^{i} \neq \varnothing$ for all $i=1, \cdots, n$. But $P_{2} \cap P_{0} \neq \varnothing$ and $Q_{2} \cap P_{0} \neq \varnothing$ implies $Q_{2}^{1} \cap P_{0}^{1} \neq \varnothing$ and $P_{2}^{1} \cap P_{0}^{1} \neq \varnothing$. Hence, we may assume, by symmetry, that $P_{2}^{1} \cap P_{1}^{1} \neq \varnothing$ and $P_{2}^{1} \cap Q_{1}^{1} \neq \varnothing$. But then $P_{2} \cap Q_{1}=\varnothing$ implies that $P_{2}^{i} \cap Q_{1}^{i}=\varnothing$ for some $i>1$; and, by symmetry, we may assume that $i=2$.

Moreover, $P_{2}^{2} \cap Q_{2}^{2} \neq \varnothing$ and $Q_{2}^{2} \cap Q_{1}^{2} \neq \varnothing$. But $P_{2}^{2}$ and $Q_{1}^{2}$ are separated by an open interval $I_{1}$. Hence, $Q_{2}^{2}$ contains $I_{1}$, since $Q_{2}^{2}$ is connected. In fact, if $R$ belongs to $C, R^{2} \cap P_{2}^{2} \neq \varnothing$ and $R^{2} \cap Q_{1}^{2} \neq \varnothing$, then $R$ contains $I_{1}$; so, $R^{2} \cap Q_{2}^{2} \neq \varnothing$. Further, if $R^{1} \cap P_{0}^{1} \neq \varnothing$, then $R^{1} \cap Q_{2}^{1} \neq \varnothing$, since both $R^{1}$ and $Q_{2}^{1}$ contain the right-hand endpoint of $P_{0}^{1}$.

Thus, we have shown that, if we are in the plane, any $R$ in $C$ intersecting $P_{0}, P_{2}$, and $Q_{1}$ must intersect $Q_{2}$ as well, contradicting the requirement that exactly two rectangles of $C$ do not intersect $Q_{2}$.

In $n>2$ dimensions, we suppose that $C$ has at least $2 n+4$ members, the first five of which are $P_{0}, P_{1}, Q_{1}, P_{2}$, and $Q_{2}$ as above. We then iterate the above argument, obtaining a list of pairs of members of $C: P_{3}, Q_{3}$; $P_{4}, Q_{4} ; \cdots ; P_{n+1}, Q_{n+1}$, such that each $P$ and $Q$ of a pair intersect each other and all the preceding members of the list except the preceding $Q$ or $P$ respectively. Thus, $Q_{n+1}$ intersects all others on the list except $P_{n}$. Further, we may assume, by symmetry that for each $i=2, \cdots, n-1$, $P_{i+1}^{i+1}$ and $Q_{i}^{i+1}$ are separated on the $(i+1)$ th coordinate axis by an open interval $I_{i}$. Hence, and member $Q_{0}$ of $C$ not already accounted for on the 
preceding list must intersect $P_{n+1}$. For, $P_{n+1}^{i}$ contains the intervals $I_{i}$ by virtue of $P_{n+1}$ intersecting $P_{i}$ and $Q_{i-1}, 2 \leqq i \leqq n$; and $Q_{0}$ also has this property. Moreover, both $P_{n+1}^{1}$ and $Q_{0}^{1}$ contain the right-hand endpoint of $P_{0}^{1}$. But this contradicts the requirement that $P_{n+1}$ intersect all but two members of $C$, completing the proof. Q.E.D.

Proof of TheOrem 1. To show the existence of dual necklaces claimed in the theorem, proceed as follows: A realization of $2 n+3$ (hypercubic) spheres in $S^{n}, n \geqq 3, P_{0}, P_{1}, Q_{1}, \cdots, P_{n+1}, Q_{n+1}$ is obtained by following the pattern discussed in the previous proof, except that we require $P_{n+1}$ and $Q_{n+1}$ to be disjoint. We need only give their projections on the $n$ coordinate axes. (See Table I.)

The symbol "*", on an axis means that the projections of spheres not explicitly accounted for on that axis take position *. Observe that on the $x_{1}$-axis $P_{0}$ does not intersect $Q_{1}$ or $P_{1} ; P_{2}$ does not intersect $Q_{1}$. On the $x_{n}$-axis, $P_{n+1}$ does not intersect $Q_{n+1}$ or $Q_{n} ; P_{n-1}$ does not intersect $Q_{n}$. Finally, on all the remaining $x_{i}$-axes, $P_{i+1}$ does not intersect $Q_{i+2}$, or $Q_{i} ; P_{i-1}$ does not intersect $Q_{i}$. However, all other intersections do occur, thus yielding the required realizations of $2 n+3$ members.

Similarly, Table II gives a realization of $2 n+2$ spheres in the space $S^{n}, n \geqq 3$.

It is clear that a realization in one space gives realizations in all higher dimensional $S^{n}$. Thus, we will be finished when we give realizations of size six and seven in $S^{2}$ and $S^{3}$, respectively. For the latter, we take spheres with centers at $(-1,8,8),(10,2,8),(3,5,3),(6,8,1),(12,6,8)$, $(6,12,10),(6,8,12)$. For the former, take spheres with centers at $(2,9)$, $(5,12),(8,14),(12,5),(8,2),(15,8)$. In each case let the spheres have radii equal to four. Q.E.D.

Definition 3. A dual necklace of $k$ spheres in $S$ removes axis $x_{i}$ if it contains spheres $P$ and $Q$ such that $P^{i} \cap Q^{i}=\varnothing$.

LEMMA 2. Each dual necklace of $k$ spheres in $S, n \geqq 2$, removes at least $r$ axes, where $2 r+1 \leqq k \leqq 2 r+3$.

Proof. Immediate by the proof of Lemma 1.

LEMMA 3. There does not exist a collection of $k$ rectangular parallelopipeds (n-dimensional, with edges parallel to the coordinate axes) satisfying condition 1 for $k>4 n$ in $R^{n}, n \geqq 1$.

Proof. We will call the members of such collections "spheres" for the sake of brevity. Now, each multi-dual necklace of spheres (abbreviated "'mdn") may be conceived as a finite set of dual necklaces (abbreviated "dn') such that each sphere of one dn intersects every sphere in any other 


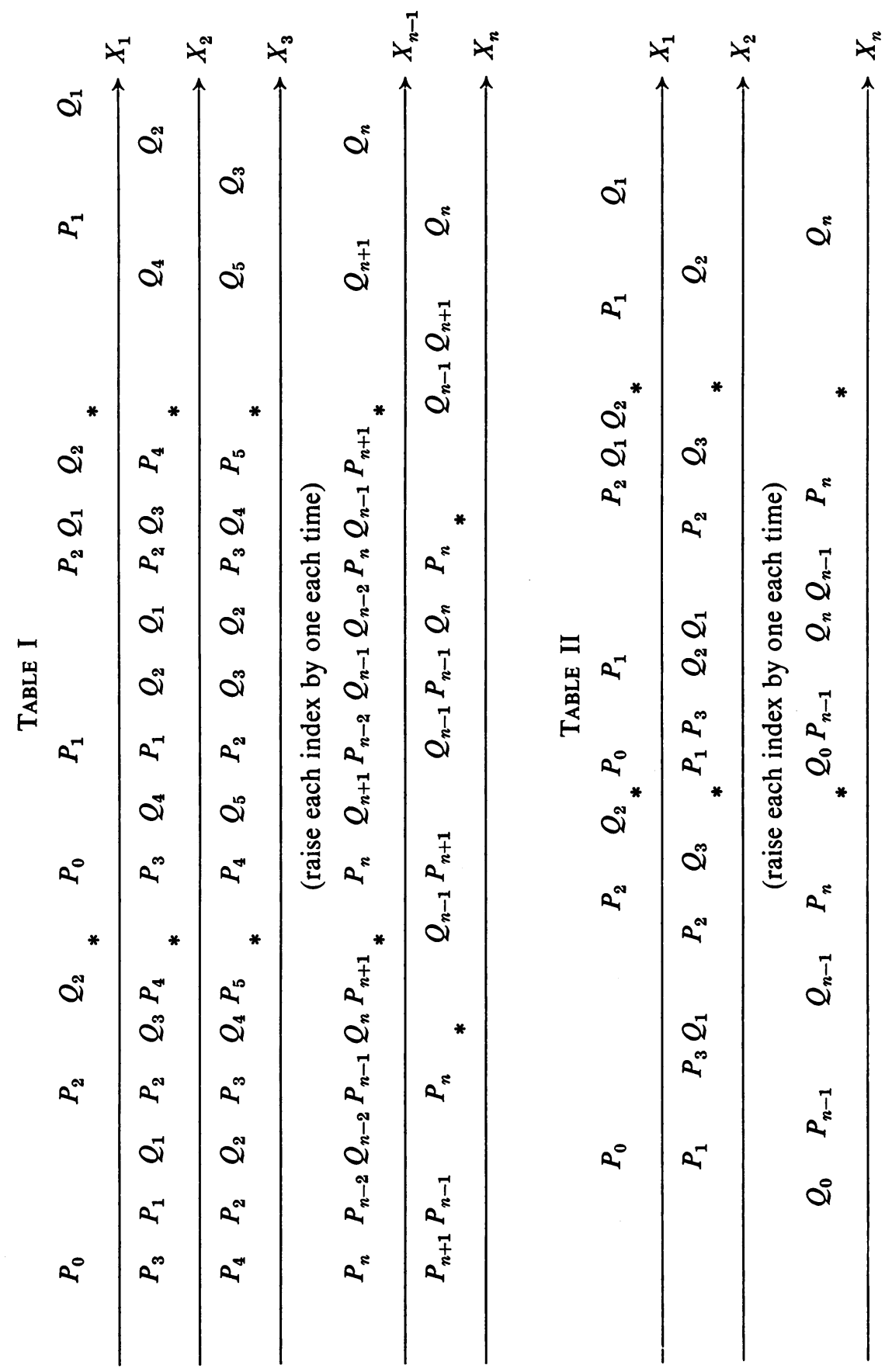


$\mathrm{dn}$ in the set. This is seen by considering the abstract graph formed by connecting spheres which do not intersect and obtaining thereby a finite set of disjoint cycles. Each sphere will be in a finite cycle, by the proof of Lemma 1 . Now, letting $[k]$ denote a dn of $k \geqq 3$ spheres, a set of $j \geqq 1$ distinct dn's forming an mdn will be denoted by $\left[k_{1}, k_{2}, \cdots, k_{j}\right]$. We claim that there do not exist mdn's of types $\left[k_{1}, \cdots, k_{j}\right]$ in $S^{n}$ for $j>n$. For, two different dn's in an mdn cannot remove the same axis; and each $\mathrm{dn}$ removes at least one axis. Hence, any $\operatorname{mdn}\left[k_{1}, \cdots, k_{j}\right]$ removes at least $j$ different axes.

Next, we claim that there is an mdn of type $\left[k_{1}, \cdots, k_{n}\right]$, where $k_{i}=4$ for each $i=1, \cdots, n$ in $S^{n}$. Moreover, $4 n>k_{1}^{\prime}+\cdots+k_{j}^{\prime}$ where $\left[k_{1}^{\prime}, \cdots, k_{j}^{\prime}\right]$ is any other mdn of the kind under consideration.

For the existence, let the $k$ be defined by the following four spheres, each having a radius of four; the $i$ th coordinate of their centers is 0 , except for the $i=j$ th, where the coordinates are $-6,-5,5$, and 6 . Intersection properties are easily verified on the coordinate axes.

Secondly, let $\left[k_{1}, \cdots, k_{j}\right]$ be an mdn of type other than $\left[4_{1}, \cdots, 4_{n}\right]$ in $S^{n}$. Hence, some $k_{i} \neq 4$, say $k_{j}$, or $j<n$. Consider $\left[k_{1}, \cdots, k_{j-1}\right], j>1$. (The case $j=1$ is trivial.) If $2 k+1 \leqq k_{j} \leqq 2 k+3$, then [ $\left.k_{j}\right]$ removed at least $k$ axes, say $x_{1}, \cdots, x_{k}$, upon which the projections of the remaining spheres all intersect. That is, on these axes there are intervals $I_{1}, \cdots, I_{k}$ which the projections of every sphere of $\left[k_{1}, \cdots, k_{j-1}\right]$ contain. On the remaining axes $x_{k+1}, \cdots, x_{n}$ the projections of any sphere $P$ of $\left[k_{j}\right]$ intersects the projections of every sphere of $\left[k_{1}, \cdots, k_{j-1}\right]$. But $4 k>$ $2 k+3$ if $k>1$. Using these facts, it is not too hard to form an mdn of type $\left[k_{1}, \cdots, k_{j-1}, k_{j}^{\prime}, \cdots, k_{n}^{\prime}\right]$ where $k_{i}^{\prime}=4$. (We leave this to the reader.) This proves the second claim. Q.E.D.

Proof of THeORem 2. By Lemma 3, only the construction of the required mdn's remains and, in fact, only those which are of size between $4 n$ and $4(n-1)$; for, the others are obtained inductively from lower dimensions. We may obtain these by combining $(n-1)$ dn's of type [4] with one of the type [3], and $(n-2)$ of types [4] with one of types [6] and [5], respectively. Again, we leave the details to the reader (e.g., use the construction devices employed above).

REMARK 1. The term "dual necklace" is derived from the fact that the dual of the theorem of Sanders, concerning shortest rectilinear polygons, yields what intuitively corresponds to a necklace. Both theorems are in fact true, with the obvious modifications, in any geodesic metric space, including, of course, $S^{n}$ (see [1] for proof).

REMARK 2. The arguments given in Lemmas 1,2 , and 3 work equally well if instead of parallelopipeds in $R^{n}$ we consider parallel, centrally symmetric, convex $2 n$-gons in the plane. (These are the spheres of certain 
normed linear spaces.) Two of these will intersect if and only if the connected regions formed by extending both pairs of corresponding parallel sides intersect. Therefore, we need only set up a pencil of axes in the plane, that is, lines perpendicular to the sides of the $2 n$-gons. Then, in the relevant proofs, use the intersections of the strip-like regions with the corresponding perpendicular axis. Thus, sets of $k$ parallel, centrally symmetric $2 n$-gons which satisfy (1) and (2) do not exist if $k>2 n+3$; those satisfying condition (1) do not exist if $k>4 n$.

REMARK 3. We close with the following question (raised by the referee): Given a dual or multi-dual necklace of $k$ subsets, $k$ satisfying certain bounds, under what conditions is it a necklace of some metric space spheres?

ACKNOWLEDGEMENTS. Some of the results appearing in this paper (viz., Theorem 1) are contained in the author's doctoral dissertation in mathematics at the City University of New York, written under the direction of Professor Fred Supnick (unpublished).

\section{REFERENCES}

1. David Sanders, On extremal circuits, Doctoral Dissertation, City University of New York, 1968 (unpublished).

Department of Mathematics, Montclair State College, Upper Montclair, NEW JERSEY 07043 